\title{
Coherent transfer functions and extended depth of field
}

\author{
M. Villiger*a ${ }^{\mathrm{a}}$ C. Pache ${ }^{\mathrm{a}}$, Rainer A. Leitgeb ${ }^{\mathrm{b}}$, Theo Lasser ${ }^{\mathrm{a}}$ \\ ${ }^{a}$ Laboratoire d'Optique Biomédicale, Ecole Polytechnique Fédérale de Lausanne, CH-1015 Lausanne, \\ Switzerland; \\ ${ }^{\mathrm{b}}$ Center of Biomedical Engineering and Physics, Medical University of Vienna, Waehringerstr. 13, A-1090 \\ Vienna, Austria
}

\begin{abstract}
To preserve the speed advantage of Fourier Domain detection in Optical Coherence Microscopy (OCM), extended depth of field (DOF) is needed. To assess and improve the DOF and the lateral resolution, we analyzed the coherent transfer function (CTF) of OCM. In the spectral domain detection, each wavelength has its own specific CTF, sampling a different part of the object's spatial frequency spectrum. For classical optics and increasing numerical apertures these regions start to overlap and bend, which limits the depth of field. Bessel-like beams produced by axicon lenses circumvent these detrimental effects, but introduce side lobes. Decoupling the detection and the illumination apertures gives more flexibility in engineering a CTF and optimizes the lateral resolution and the DOF at the same time all while reducing these side lobes.

We evaluated different combinations of Gaussian and Bessel-like illumination and detection optics. Using Bessel-like beams as well in the illumination as in the detection paths, but with different side-lobe radii, we obtained a lateral resolution of $2 \mu \mathrm{m}$ invariant over an extended depth of field of more than $300 \mu \mathrm{m}$, at a signal penalty of only $12 \mathrm{~dB}$ compared to classical Gaussian optics.
\end{abstract}

Keywords: extended depth of field, Optical Coherence Microscopy, Axicon, Bessel-like beams, Coherent Transfer Function

\section{INTRODUCTION}

Fourier domain optical coherence microscopy (FDOCM) attempts to combine the advantages of FDOCT such as imaging speed, detection sensitivity [1,2] and the availability of signal phase [3] with the high lateral resolution of microscopy [4]. It has the potential to access the structure and functionality of the cell-organism in vivo and visualize time dependent events [5].

In FDOCT the depth resolution is defined by the coherence length of the light source and is decoupled from the lateral resolution. The available depth range is defined by the spectral width of an individual pixel on the spectrometer and is in the order of a few millimeters. On the other hand, the lateral resolution is defined by the employed optics and scales with 1/NA (numerical aperture). The resulting depth of field (DOF) is given by the Rayleigh-criterion and is proportional to $1 / \mathrm{NA}^{2}$. The optical sectioning of this short DOF is already pronounced at even small NAs and reduces significantly the depth range. For higher NA it can even overcome the $\mathrm{z}$ resolution provided by the coherence length, and hampers FDOCT parallel depth probing.

Several works $[6,7,8,9]$ report on employing axicon lenses or phase filters to obtain Bessel-like focal volumes. Such Bessel-like beams provide a strong central lobe, extending over a long axial range. This central lobe is flanked by side lobes, which all carry approximately the same amount of energy as the central lobe and can degrade the image quality. We have introduced extended focus optical coherence microscopy (xfOCM) [10], where we decoupled the detection aperture from the illumination and used classical low NA Gaussian detection optics. This decoupling revealed essential for the image quality, since the side lobes where significantly suppressed. Still, outside the DOF of the detection optics the signal dropped and the side lobes emerged. Also, the detection still depends on the NA and cannot be scaled to higher NA easily.

*martin.villiger@epfl.ch; phone +41 2169377 73; fax +41 2169378 20; 
To improve the understanding of the imaging performance, we analyzed the coherent transfer function (CTF) of OCM $[11,12]$. Here, we theoretical and experimentally investigated the performance of a setup with classical Gaussian optics (illumination and detection Gaussian with $1.6 \mu \mathrm{m}$ and $2.5 \mu \mathrm{m}$ waist in the focal plane respectively), a Bessel illumination with Gaussian detection configuration (Bessel illumination with first zero at $1.9 \mu \mathrm{m}$, Gaussian detection with $2.5 \mu \mathrm{m}$ waist), and a new Bessel illumination and Bessel detection mode, where the radii of the illumination and detection Bessel side lobes are different (Bessel illumination with first zero at $1.9 \mu \mathrm{m}$, Bessel detection with first zero at $3.9 \mu \mathrm{m}$ ).

\section{THEORY}

The intensity pattern recorded on the CCD line camera of the spectrometer as function of the wave number $k$ and the sample's lateral scanning position $\boldsymbol{r}=(x, y)$ is expressed as

$$
I(\boldsymbol{r}, k)=\left|S_{r}(k)\right|^{2}+|S(\boldsymbol{r}, k)|^{2}+S(\boldsymbol{r}, k) S_{r}^{*}(k)+S^{*}(\boldsymbol{r}, k) S_{r}(k),
$$

where $S_{r}$ is the reference term and $S$ the sample signal. According to the first order Born approximation, the sample term $S$ can be written

$$
S(\boldsymbol{r}, z, k) \propto A(k) \int_{\boldsymbol{r}^{\prime}, z} U_{i l l}\left(\boldsymbol{r}^{\prime}, z^{\prime}, k\right) U_{\mathrm{det}}\left(\boldsymbol{r}^{\prime}, z^{\prime}, k\right) \eta\left(\boldsymbol{r}-\boldsymbol{r}^{\prime}, z-z^{\prime}\right) d \boldsymbol{r}^{\prime} d z^{\prime} .
$$

Here, $\mathrm{A}(\mathrm{k})$ is the spectral envelope, $U_{\text {ill,det }}(\boldsymbol{r}, z, k)$ are the illumination and detection field modes and $\eta(\boldsymbol{r}, z)$ is the sample structure. For the following demonstration, the sample is scanned not only in the lateral directions $r$, but also along $z$, as in confocal microscopy. Defining the CTF in function of $\boldsymbol{q}, s-$ the spatial frequency Fourier pairs of $\boldsymbol{r}, z$ - as

$$
\operatorname{CTF}(\boldsymbol{q}, s, k)=C T F_{i l l} *_{\boldsymbol{q}, s} C T F_{\mathrm{det}}=F T_{\boldsymbol{r}, z}^{-1}\left\{U_{\text {ill }} \cdot U_{\text {det }}\right\}
$$

we can now write the signal as the product of the sample spatial frequency spectrum, multiplied by the CTF and the spectral envelope:

$$
\tilde{S}(\boldsymbol{q}, s, k) \propto A(k) C T F(\boldsymbol{q}, s, k) \tilde{\eta}(\boldsymbol{q}, s) .
$$

According to the theory of generalized apertures [11] the CTFs of the illumination and the detection correspond to sphere caps of radii $k$. The angular field distribution on the caps corresponds to the angular field distributions of each mode (illumination and detection) in the principle plane of the sample objective. The total CTF is the convolution of the illumination and the detection cap and defines in general a volume in the spatial frequency domain. Depending on the optics, this volume scales and shifts in function of $k$.

To return from $\boldsymbol{q}$ and $s$ to $\boldsymbol{r}$ and $z$, the Fourier transform is calculated:

$$
S(\boldsymbol{r}, z, k) \propto A(k) \iint_{\boldsymbol{q}}\left(\int_{s} C T F(\boldsymbol{q}, s, k) \tilde{\eta}(\boldsymbol{q}, s) e^{-i 2 \pi s z} d s\right) e^{-i 2 \pi \boldsymbol{r} r} d \boldsymbol{q} .
$$

In FDOCT, the scanning along $z$ is omitted, and we can set $z=0$ in the above expression. For each pixel of the spectrometer, the interference term acquired after a two-dimensional scan corresponds to the product of the sample spectrum with the pixel's CTF, projected along $s$. As long as the CTFs of the different pixels give access to distinct volumes in the spatial frequency spectrum, this spatial frequency information is correctly sampled as a function of $k$ and can be retrieved by Fourier transformation from $k$ to $z$. If however the individual CTFs start to overlap and bend, correct sampling is no longer assured, resulting in loss of information, which results in a limited depth of field. 
In the following, for sake of simplicity, we ignore the scaling of the CTF with respect to $\mathrm{k}$, and pretend it is merely shifted axially along s. In this case an estimate of the OCT PSF can be found by taking the Fourier transform of the CTF and adding the coherence gating of the source spectral width.

Figure 1 shows the CTFs for different illumination and detection combinations for three $k$ in the top row. The lower row gives an impression of the expected tomogram for point-like idealized scatterers aligned along the optical axis. All the CTFs have roughly the same lateral support, resulting in comparable lateral resolutions in the focal plane. The bending and the width of the CTF along $s$ determine the DOF. The Gaussian-Gaussian configuration results in the shortest DOF. The Bessel-Gauss combination reduces the support width along s of the CTFs, resulting in an increased DOF. Still, the CTF is curved, producing lateral blurring of the detection volume and hence the appearance of strong side-lobes in these out of focus regions. The CTF for the Bessel illumination, combined with Bessel detection with altered side-lobe spacing, shows a near-ideal CTF, with no curvature and a very narrow support along s. The side-lobes are also reduced in amplitude.

Figure 2 on the left hand side shows the projection of the CTF of the central wavelength along S to give an impression of the transmitted lateral spatial frequencies. The Gaussian detection produces a smooth CTF, even in case of Bessel-like illumination. The Bessel-Bessel configuration however results in a rugged transmission spectrum and low lateral spatial frequencies are nearly completely blocked, producing a dark-field effect. The CTF in this case has no curvature and a short s-support, but the non-smooth transmission profile has some detrimental effects on the PSF.

Figure 3 shows the radial PSF of the different configurations in comparison. The side-lobes from the Bessel-like patterns are evident. The Gaussian detection suppresses the higher order side-lobes very efficiently within a limited depth range. Outside this range, signal from the side-lobes may be detected. Although the Bessel-Bessel configuration has prominent side-lobes, they are unaltered along a long axial range, and stay at least $20 \mathrm{~dB}$ below the central peak.
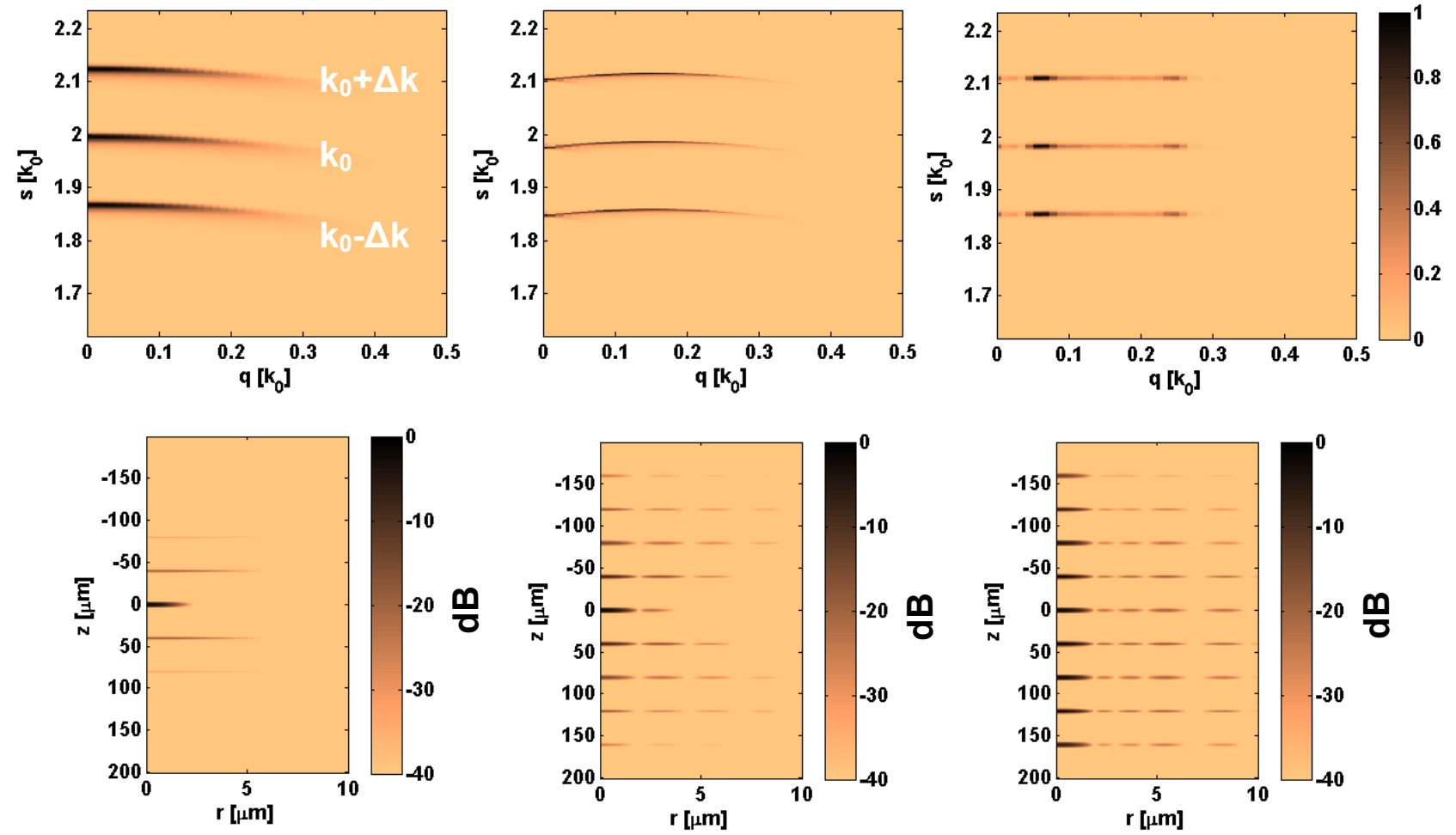

Figure 1: Top row: CTFs for different illumination and detection combinations (Gaussian-Gaussian, Bessel-Gaussian, Bessel-Bessel', from left to right) for three different pixels $\left(\mathrm{k}_{0}-\Delta \mathrm{k}, \mathrm{k}_{0}, \mathrm{k}_{0}+\Delta \mathrm{k}\right)$. Bottom row: Corresponding simulated tomograms for idealized point-like scatterers aligned along the optical axis. 

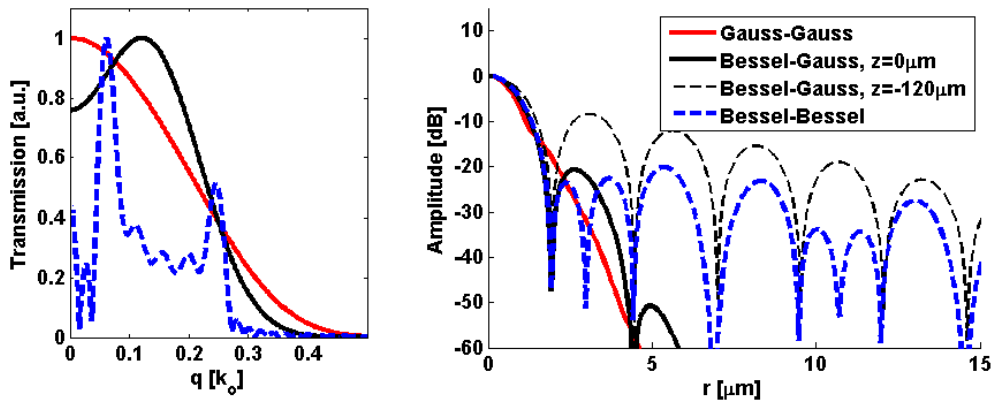

Figure 2: The left hand side shows the projection of the CTF for the central wavelength along s to give an impression of the transmitted lateral spatial frequencies. The right hand side figure displays the radial shape of the PSF.



Figure 3: Layout of the setup.

\section{EXPERIMENTAL WORK}

We implemented the different illumination and detection modules into our scanning xfOCM setup, displayed in figure 3. The broad bandwidth light source (Ti:Sapphire) with center wavelength at $800 \mathrm{~nm}$ and a spectral bandwidth of $135 \mathrm{~nm}$ is collimated $\left(\mathrm{L}_{\mathrm{C}}, \mathrm{f}=8.2 \mathrm{~mm}\right)$ and split by means of a 50:50 beam splitter into reference and sample arm. In the sample arm the collimated beam passes either an axicon lens (Asphericon, $176^{\circ}$ apex angle) for the Bessel-Gauss and BesselBessel case, or a normal lens $(\mathrm{f}=80 \mathrm{~mm}$ ) for the Gauss-Gauss combination. The illumination is relayed through the scanning system $\left(\mathrm{L}_{\mathrm{R}}\right)$, deflecting the beam in both lateral directions, to the tube lens $\left(\mathrm{L}_{\mathrm{T}}, \mathrm{f}=164 \mathrm{~mm}\right)$ and the sample objective ( $\mathrm{L}_{\mathrm{S}}, 10 \mathrm{x}$ Zeiss Plan Neofluar). Another beam splitter decouples the detection and adds the reference signal. The detection optics consist either of lens $\mathrm{L}_{\mathrm{T}}(\mathrm{f}=164 \mathrm{~mm}$, Bessel-Gauss and Gauss-Gauss) or an axicon (Del Mar, 179 , Bessel-Bessel) and an objective lens $\left(\mathrm{L}_{\mathrm{D}}\right)$ coupling the signal into the detection fiber (10x Zeiss Plan Neofluar with $\mathrm{L}_{\mathrm{T}}$, 20x Zeiss Plan Neofluar with the axicon).

The interference signal is then recorded with a spectrometer consisting of a transmission grating (1200 lines $/ \mathrm{mm})$, a camera objective $(\mathrm{f}=135 \mathrm{~mm})$ and a line detector with 2048 pixels set to a frame rate of $20 \mathrm{kHz}$. The spectrometer resolution is $\delta \lambda=0.08 \mathrm{~nm}$ allowing for a maximal system depth range of $1.97 \mathrm{~mm}$ in air.

To compare the performance of the different combinations, figure 4 shows en face images of an orange slice from different depths (in focus, and $150 \mu \mathrm{m}$ in front (-) and behind (+) the focal plane, where "in focus" is the depth of the strongest signal). The in-focus image of the Gauss-Gauss setup shows a clear tomogram with high sensitivity. Outside the short DOF the signal is however severely blurred. The Bessel-Gauss image has a significantly enlarged DOF and produces high contrast also outside the Rayleigh range of the detection mode. The detrimental effect of the side-lobes is however noticable. In case of a Bessel beam, the energy of the incoming beam is distributed over the numerous side lobes. The power passing in the central lobe is significantly reduced compared to the Gaussian beam, where $83 \%$ of the beams energy travels through the waist radius. To compensate for this effect, we increased the illumination power to yield the same field amplitude in the focal point. Although the peak amplitudes are equal to the Gaussian illumination, a larger number of photons irradiates the sample in this case.

While it is possible to compensate for this efficiency loss on the illumination side, detection with a Bessel-like beam is inherently reduced, and cannot be compensated for. A signal penalty of $12 \mathrm{~dB}$ is the price to pay for the invariant resolution over a range of more than $300 \mu \mathrm{m}$, showing clearly defined structure over the whole imaged range. 


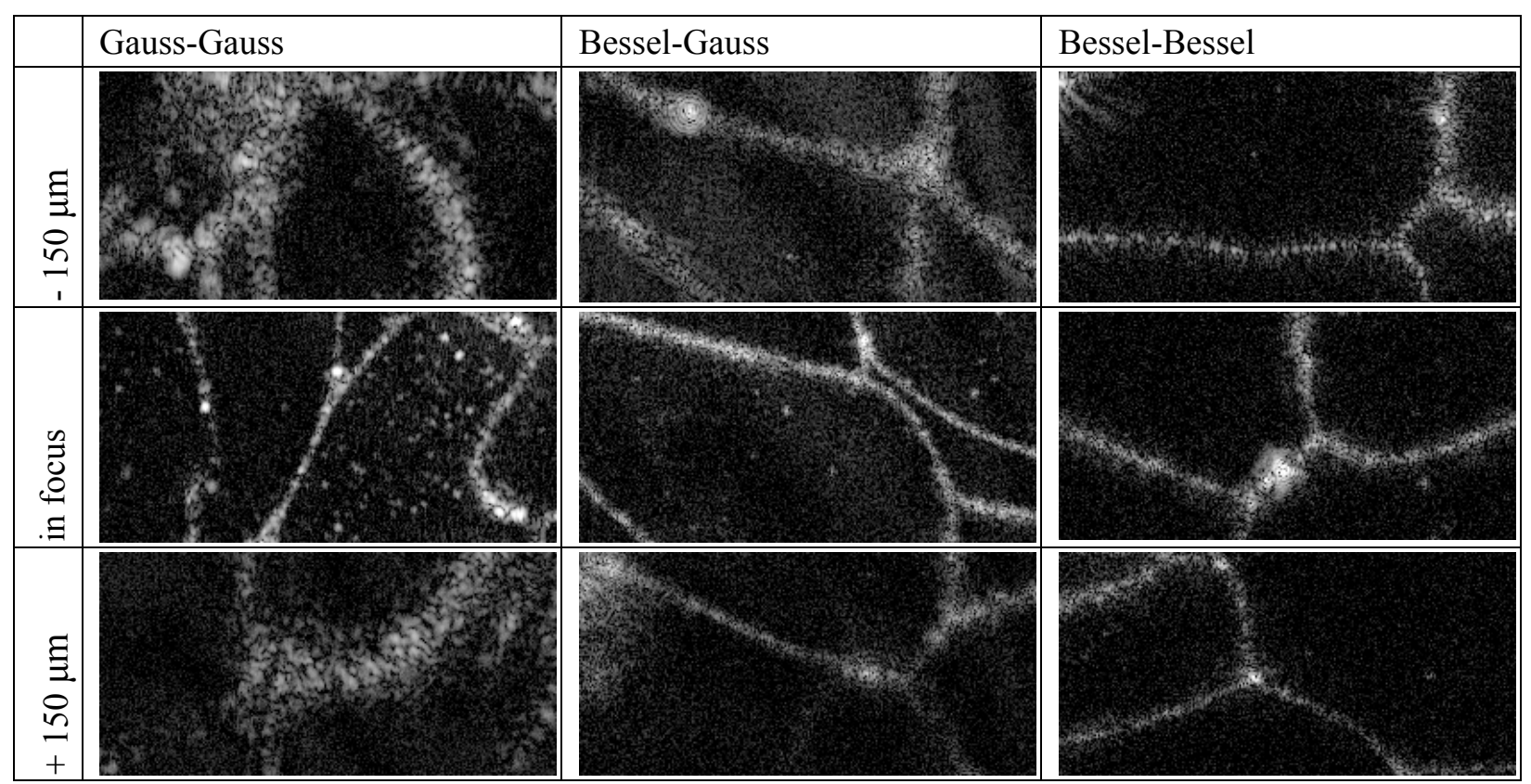

Figure 4: En face images at various depths of a slice of an orange (30dB of dynamic range, same absolute gray scale).

\section{CONCLUSION}

The analysis of the CTF for different illumination and detection combinations improves the understanding of image formation in FDOCM and helps to engineer optics with an extended depth of field. Decoupling the illumination and detection apertures provides more flexibility in optimizing the system's CTF. The combination of two different Besselbeams for the illumination and detection provides currently the best compromise for high lateral resolution of $\sim 2 \mu \mathrm{m}$, reduced side-lobes and a large DOF of more than $300 \mu \mathrm{m}$ at a sensitivity reduced by $12 \mathrm{~dB}$ compared to the confocal Gaussian-Gaussian case.

\section{REFERENCES}

[1] Leitgeb, R., Hitzenberger, C., K. and Fercher, A., F. , "Performance of fourier domain vs. time domain optical coherence tomography," Optics Express 11, 889-894 (2003).

[2] Nassif, N. et al. " "In vivo human retinal imaging by ultrahigh-speed spectral domain optical coherence tomography," Opt. Lett. 29, 480-482 (2004).

[3] Choma, M., A., Ellerbee, A., K., Yang, C., H., Creazzo, T., L. and Izatt, J., A. , “Spectral-domain phase microscopy,” Opt. Lett. 30, 1162-1164 (2005).

[4] Izatt, J., A., Hee, M., R., Owen, G., M., Swanson, E., A. and Fujimoto, J., G. , “Optical Coherence Microscopy in Scattering Media," Opt. Lett. 19, 590-592 (1994).

[5] Huang, S., W., Aguirre, A., D., Huber, R., A., Adler, D., C. and Fujimoto, J., G. , "Swept source optical coherence microscopy using a Fourier domain mode-locked laser," Optics Express 15, 6210-6217 (2007).

[6] Ding, Z., H., Ren, H., W., Zhao, Y., H., Nelson, J., S. and Chen, Z., P. , "High-resolution optical coherence tomography over a large depth range with an axicon lens," Opt. Lett. 27, 243-245 (2002).

[7] Liu, L., B., et al. , "Superresolution along extended depth of focus with binary-phase filters for the Gaussian beam," J. Opt. Soc. Am. A-Opt. Image Sci. Vis. 25, 2095-2101 (2008).

[8] Lee, K., S. and Rolland, L., P. , "Bessel beam spectral-domain high-resolution optical coherence tomography with micro-optic axicon providing extended focusing range," Opt. Lett. 33, 1696-1698 (2008).

[9] Tan, K., M., et al. , "In-fiber common-path optical coherence tomography using a conical-tip fiber,” Optics Express 17, 23752384 (2009).

[10] Leitgeb, R., A., Villiger, M., Bachmann, A., H., Steinmann, L. and Lasser, T. , "Extended focus depth for Fourier domain optical coherence microscopy," Opt. Lett. 31, 2450-2452 (2006).

[11] McCutchen, C., W. , "Generalized Aperture + 3-Dimensional Diffraction Image," Journal of the Optical Society of America 54, 240-\& (1964).

[12] Sheppard, C., J., R., Gu, M. and Mao, X., Q., "3-Dimensional Coherent Transfer-Function in a Reflection-Mode Confocal Scanning Microscope,” Opt. Commun. 81, 281-284 (1991). 